\title{
La inmersión y la enseñanza bilingüe en el País Vasco
}

\author{
Josu Sierra
}

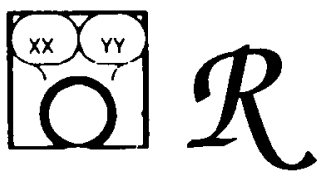

En este artículo se hace un balance de los tres modelos alternativos en la enseñanza de segunda lengua en el País Vasco: $A$ (como simple asignatura), $B$ (inmersión parcial en L2) y $D$ (inmersión total temprana). La claridad de la exposición y de los hechos presentados permite llegar a conclusiones que pueden mejorar la actual enseñanza de la L2.

\section{LA INMERSION EN LA ADQUISICION DE UNA SEGUNDA LENGUA Y LOS MODELOS DE ENSEÑANZA BILINGÜE EN EL PAIS VASO}

Hace ya algunos años que comenzamos a oír en el País Vasco la palabra «inmersión» como sistema de aprendizaje de una segunda lengua. Se trataba de un programa canadiense que obtuvo gran éxito y resonancia internacional. Por aquel entonces, muchos no sabíamos muy bien en qué consistía la citada inmersión pero decían que se trataba de utilizar desde un principio la segunda lengua (L2) a todos los efectos, algo parecido - pensamos- al programa tipo «D» que las ikastolas practicaban en las zonas más castellanoparlantes del País Vasco. Hoy día, los programas de inmersión no han perdido un ápice de su «aureola» inicial ni de su vigencia, al contrario, permanecen como un valor seguro e incluso pujante obtenido de sucesivas experiencias positivas y de una cada vez más abundante investigación acerca de sus resultados en diferentes países y con diversas lenguas a lo largo de todo el mundo. Al mismo tiempo, otros programas más «débiles» (no tan intensivos) de aprendizaje de una segunda lengua han sido puestos en cuestión a la luz de su eficacia (Cummins, 1989), replicando una vez más la situación que llevó a los padres canadienses de St. Lambert a plantearse la superación del método clásico de aprendizaje de un segundo idioma.

El caso catalán es un exponente claro de la pujanza antes citada y hoy día son ya muchos miles los niños que siguen un programa de inmersión en Catalunya, con resultados palpables y un éxito que casi ha llegado a sor- 
prender a sus propios promotores (Artigal, 1989). Teniendo en cuenta la costumbre que ha existido en Euskadi de importar de Catalunya más de una moda en lo que al tratamiento de la Educación Bilingüe se refiere, no sería de extrañar que los programas de inmersión hicieran furor tarde o temprano, siempre que las actitudes favorables existentes actualmente hacia la educación bilingüe se mantengan. Los programas de aprendizaje de y en Euskera, vigentes hoy día en la Comunidad Autónoma Vasca, llamados "modelos de enseñanza bilingüe», son el A el B y el D. El modelo A no utiliza la L2 como lengua de instrucción, sino que la limita a mera asignatura. El B, propugna una inmersión parcial con porcentajes de L2 variables a lo largo de la educación básica. Oficialmente la lectoescritura y las matemáticas se imparten en $\mathrm{L} 1$. El modelo $\mathrm{D}$, pensado para vascoparlantes nativos, es un programa de instrucción total euskera, siendo ésta la L2 para los sujetos castellanoparlantes escolarizados en él. Estos modelos, han tenido un rodaje y están produciendo unos resultados que hace ya algún tiempo se empezaron a evaluar (Gabiña, Gorostidi, Iruretagoiena, Olaziregi y Sierra, 1986). Estos atribuyen una desigual eficacia a los diferentes «modelos" a la hora de conseguir que los sujetos de los diversos programas de enseñanza bilingüe (si es que así puede considerarse al A), dọminen las dos lenguas al término de su escolaridad básica.

En el más reciente de los estudios publicados (Olaziregi y Sierra, 1989), se evidencian unos peores resultados de L2 en los modelos más «débiles» y próximos al tratamiento de esta lengua como mera asignatura, poniendo en duda su "rentabilidad" para conseguir el fin para el que fueron creados. Concretamente el modelo A y algunos tipos de B (modelo heterogéneamente aplicado, con variaciones metodológicas), obtienen niveles bajos en euskera, lo que puede propiciar el desencanto de comprobar que tras años de aprendizaje, el nivel alcanzado es totalmente insuficiente, acarreando una desmotivación de los sujetos entre otras consecuencias.

Respecto a la cuestión de si en el País Vasco se practica o no la inmersión a través de los modelos actualmente en marcha, puede decirse que, la mayoría de ellos, no coinciden plenamente con la metodología practicada en Canadá, en ninguna de sus modalidades ni con la distribución de tiempos de instrucción en una lengua y otra. No debiera confundirse con el modelo tipo "D", de escolarización total en L2, ya que este modelo estaba pensado para sujetos vascoparlantes. A pesar de ello se ha extendido a muchos castellanoparlantes, para los que el euskera se convertía en segunda lengua, por lo que se ha debido adaptar de forma sustancial a la nueva realidad. Oficialmente se sigue considerando que existe un solo modelo D a todos los efectos y sigue siendo un programa de instrucción total en euskera. Como veremos, el más homologable a una inmersión parcial es el modelo B.

\section{CARACTERISTICAS DE LA INMERSION}

Estos programas de aprendizaje en una segunda lengua, nacieron en medio del proceso de recuperación del francés en Quebec. Los hijos de algunos habitantes anglófonos de esta provincia, muy motivados en el proceso de bilingüización, seguían programas de francés de escaso rendimiento y dudoso futuro (Hamers y Blanc, 1983), por lo que los padres deseaban dar 
la oportunidad a sus hijos de convertirse en sujetos bilingües y que este nuevo estatus les reportase beneficios sin por ello perjudicar a la lengua materna ni a los resultados académicos. De este modo se ofrecía a aquellos niños la posibilidad real de integración en la cultura francófona consiguiendo un bilingüismo "aditivo" (Lambert, 1975). La experiencia piloto de St. Lambert ha sido la que ha recorrido el mundo (Lambert y Tucker, 1972) dando la vuelta a toda la investigación hasta entonces reinante de descrédito y devaluación del bilingüismo.

Antes de entrar a detallar los pormenores de los programas de inmersión, digamos que no existe una única inmersión sino varias (Hamers y Blanc, 1983). De todas ellas es la «inmersión total temprana» la más paradigmática tanto por sus características como por sus resultados. Existe sin embargo una "inmersión parcial», con menor presencia de la L2 y una «inmersión tardia» de aplicación en edades y niveles más avanzados dentro de la escolaridad normal.

Refiriéndonos a la primera de ellas, experimentada en St. Lambert, aclaremos que es una metodología apropiada para hablantes de la lengua dominante (Hamers y Blanc, 1983), es decir, para sujetos pertenecientes a colectivos cuya L1 es la lengua preponderante en ese entorno y cuyos hablantes la utilizan corrientemente en todas las funciones propias de una lengua, sin que pertenezcan a minorías étnicas marginadas o grupos lingüísticos en situación desfavorecida, lo cual implicaría una lesión para su lengua materna y llevaría, seguramente, a un proceso de aculturización. En el caso del euskera, el programa de inmersión sería aplicable a sujetos castellanoparlantes, no así a vascoparlantes (método éste ampliamente experimentado por los vascos a quienes se imponía la escolaridad en castellano hasta hace bien poco). Los sujetos pertenecientes a un grupo de lengua dominante, no tienen problema para seguir en la escuela un programa tan intensivo en L2 como la inmersión, ya que de este modo desarrollará tanto la L1 como la L2 (relativamente, como veremos), sin que ello suponga menoscabo de su lengua materna, gracias a lo que J. Cummins denomina la "competencia común subyacente" (Cummins, 1987). Muy al contrario, los aspectos positivos son evidentes.

La teoría que preside el método de inmersión es que la L2 se aprende del mismo modo que la lengua materna, se aprende utilizándola (la L2 es un instrumento para aprender cosas interesantes) y argumenta que el método no sólo no es negativo para el sujeto sino que le reporta beneficios convirtiéndole en individuo bilingüe, lo cual repercute socialmente a la identificación y aceptación del «otro* grupo lingüístico así como en un reforzamiento del pensamiento creativo y divergente, características de los individuos poseedores de un bilingüismo aditivo y enriquecedor. Acerca del beneficio (Balkan, 1979) que se deriva de estos tipos de educación bilingüe, se corre quizá el peligro de pasar alegremente de despreciar esta educación a una cierta "euforia" basándose en algunos resultados de estudios quizá no muy generalizables.

Con todo, en nuestro entorno, la investigación acerca de los programas de inmersión y sus logros no ha trascendido suficientemente a los padres, ni a los educadores, que mantienen en algunos casos actitudes reticentes fruto de la escasa información que ha recibido. El argumento de la inferioridad de los sujetos que siguen la enseñanza bilingüe en la "lengua débil» 
(Macnamara, 1966), ha sido ampliamente contestado (Cummins, 1976, 1978) por diversas investigaciones más recientes. En el País Vasco es evidente el creciente prestigio de los programas más similares a la inmersión, es decir, programas de instrucción en L2, más como fruto exclusivamente de su «buena marcha" y de la actitud general favorable hacia el euskera que del conocimiento del prestigio internacional adquirido por estos programas y de su probada validez.

Volviendo al hilo de la inmersión total temprana, recordaremos que desde el comienzo de la escolarización se utiliza solamente la L2, siendo los profesores hablantes de la L2 (bilingües, para comprender y responder al niño en su L1), manteniéndose esta situación durante tres años y posteriormente, se comenzará un programa de mantenimiento de la L1 en módulos de 30 minutos aproximadamente. Más adelante las asignaturas se repartirán unas en L1 y otras en L2, eligiendo el alumno cuáles de ellas recibirá en qué lengua (ver gráfico de porcentajes de instrucción en L1 y L2) (Genesee, Lambert y Holobow, 1986). Durante el jardín de infancia y los dos primeros cursos de la enseñanza elemental, el porcentaje de L2 en la instrucción es del $100 \%$, pasando en el curso 3 ó 4 a un $60 \%$ en L2 y al año siguiente a un $40 \%$ en esta misma lengua. Este es al menos el modelo canadiense clásico de St. Lambert, si bien, como hemos dicho, existen diversos tipos de programas de inmersión, no tan intensivos o no tan precoces, pero con resultados también inferiores (Hamers y Blanc, 1983) a los obtenidos en la total temprana, es decir, obteniendo un inferior nivel de L2 en los menos intensivos.

Veamos sin embargo, más detenidamente, el aspecto de los resultados de la inmersión: Los sujetos pertenecientes a programas de inmersión total temprana, mantienen el nivel de L1 y llegan en L2 a una competencia próxima a la de los hablantes nativos de esta lengua. Hay que precisar, no obstante, que no alcanzan el nivel de un hablante nativo en L2. Evidencian una competencia más baja en los aspectos de expresión oral y muestran un comportamiento más "pasivo" como hablantes de L2, es decir, no usan espontáneamente esta segunda lengua en sus actividades normales, o han llegado a una fase de «meseta» en su aprendizaje de L2.

Esto se ha atribuido a varios factores, entre ellos a algunos metodológicos. Cummins (1987) recoge las opiniones que achacan las dificultades de expresión de los alumnos, correspondientes a niveles superiores de programas de inmersión temprana en francés, a una pedagogía excesivamente centrada en el profesor y a una falta de «interacción comunicativa» en la metodología aplicada.

En cuanto a sus rendimientos escolares, estos alumnos obtienen notas equiparables a los sujetos que han seguido un programa normal en su primera lengua, aspecto éste que no se puede soslayar y que muchas veces provoca la desconfianza de ciertos elementos puestos a este tipo de educación bilingüe, desconfianza que, sin embargo, no se ve apoyada por la investigación existente al respecto, siempre y cuando se respeten las condiciones mínimas para la aplicación de programas de inmersión, y sobre las que hablaremos a continuación.

Las familias que toman parte en los programas de inmersión, lo hacen de un modo totalmente voluntario y poseen por tanto una motivación y una actitud positiva para con la L2 indispensables para el sujeto que toma- 
rá parte en este tipo de enseñanza bilingüe por otro lado, las condiciones psicopedagógicas exigibles, son adecuadas al programa que se va a emprender y a la metodología a utilizar: el profesorado es voluntario y fuertemente motivado, preparado para su labor, misión ésta que entraña, sin duda, delicadas responsabilidades. Su dominio de la lengua y de la didáctica de la misma deben estar fuera de toda duda, lo que exige una experiencia en este tipo de programas. A esto debe sumarse lo ya apuntado más arriba acerca de la procedencia de los sujetos a quienes se somete a este tipo de programas. La pertenencia de éstos a minorías lingüísticas o a grupos de culturas marginadas ha evidenciado unos resultados pobres en L2 y efectos perjudiciales para la L1 del sujeto, así como la aparición de actitudes que pueden llevar al desarraigo cultural de las minorias citadas (Sánchez, R. de Tembleque, 1986).

Por todo lo expuesto y retomando la cuestión de los modelos de enseñanza bilingüe del País Vasco (modelos A, B y D), es preciso reconocer que existen similitudes entre algunos de ellos y los modelos de inmersión, equiparándose incluso, por lo que parece, en sus resultados. Así, existen algunos modelos B «intensivos» (Olaziregi y Sierra, 1987) que funcionan «de facto" como modelos próximos a la «inmersión total temprana" (lecto-escritura en L2 y $100 \%$ de euskera en preescolar). El modelo D aplicado a castellanoparlantes de zonas castellanófonas, coincide también en muchas de sus características con un programa de inmersión. Pero, en el caso del $B$, le falta en primer lugar una estructuración más definida y homogénea basada en un aprovechamiento de los primeros niveles para un aprendizaje intensivo de la L2, incluyendo el aprendizaje de la lectura y escritura que permita a los niños alcanzar un nivel-umbral suficiente en su competencia en L2 para que su aprovechamiento y rendimiento escolar sea apropiado (Cummins, 1979). Además, necesitaría el reconocimiento oficial de su existencia como programa intensivo de educación bilingüe (modelos B intensivos... etc.) que pudiera proporcionarle el prestigio que internacionalmente tienen los programas de inmersión gracias a la gran cantidad de investigación realizada sobre ellos, valor éste muy importante de cara a la opinión pública y -especialmente- a los padres que son quienes deciden dónde matricular a sus hijos. Otros modelos de enseñanza bilingüe, ni siquiera considerados como tal (A), por el contrario se alejan fundamentalmente de los postulados de la inmersión así como de sus resultados, cosechando - muchas veces- fracasos y sembrando, con frecuencia, el desánimo entre los escolares, poniendo en peligro el rendimiento en las materias impartidas con un insuficiente nivel de L2 (B débiles). Este tipo de bilingüismo sí puede ser, cuando menos, no válido para el desarrollo cognitivo del sujeto.

\section{LOS MODELOS B Y D}

Detallando algo más acerca del modelo $\mathrm{B}$ de enseñanza bilingüe en vigor en el País Vasco, debemos advertir en primer lugar que su aplicación -tal y como se dijo anteriormente- difiere bastante de unos centros a otros. El diseño teórico del programa se asemeja enormemente a una «inmersión parcial» salvando las distancias entre los sistemas educativos de Canadá y el País Vasco. Esto es, la distribución del tiempo de enseñanza en 
L1 y L2 al $50 \%$ para cada lengua, con el aprendizaje de la lecto-escritura y matemáticas en L1, no es rigurosamente seguida en todas partes, pero se trata del diseño mayoritariamente aplicado en el llamado modelo $B$.

Las implicaciones de todo esto en los resultados obtenidos tanto en las destrezas en euskera como en el rendimiento académico son importantes. De este modo, el nivel de L2 alcanzado por los alumnos del programa que nos ocupa adolece muchas veces de un buen desarrollo de las destrezas expresivas en euskera tanto a nivel oral como escrito, si bien parece que obtienen un suficiente nivel de comprensión en general. (J. Sierra e I. Olaziregi, 1989.)

Tal y como se desprende de las investigaciones realizadas en Canadá (Hamers y Blanc, 1983), este tipo de «inmersión parcial» no consigue obtener un nivel de L2 suficiente para asegurar al sujeto un buen rendimiento en las materias impartidas en L2, y no por ello superan en los niveles de L1 a los sujetos que siguen un programa de inmersión total temprana. El citado handicap supone un grave lastre capaz de hipotecar el futuro de este tipo de programas excesivamente "débiles» en cuanto al «input» de L2 que el alumno recibe. Así pues, el modelo B utilizado en el País Vasco y en el que tantas esperanzas se han depositado, puede, peligrosamente a mi entender, seguir los pasos del ya criticado programa de «inmersión parcial» canadiense. Inexplicablemente, las experiencias de «inmersión total temprana», que pudieran ofrecer una alternativa si esto se confirmase, son prácticamente inexistentes en Euskadi hoy día (si descontamos los modelos B intensivos).

Quizá parte de la explicación de este fenómeno se encuentre en la confusión que rodea, en cuanto a estudios y teorización se refiere, en torno al modelo $\mathrm{D}$. Confusión en el sentido de que se denomina $\mathrm{D}$ tanto al programa de enseñanza en L2 dirigido a niños castellanoparlantes, como a aquel que ofrece una enseñanza totalmente en euskera para los niños cuya lengua materna es esta misma, lo cual supone mezclar bajo la misma sigla a programas de objetivos y metodología bien diferentes.

Esto no ocurre en Canadá, donde el francófono tiene derecho a un programa educativo en francés, mientras que los programas de inmersión están reservados a los niños que no conocen esta lengua.

Volviendo al País Vasco, para los sujetos castellanoparlantes de origen, el modelo D se convierte en un verdadero programa de *inmersión total temprana» por su intensidad (sin entrar en cuestiones metodológicas sobre el uso de lenguaje contextualizado, la interacción comunicativa... etc., vitales en la inmersión), si bien el tiempo dedicado a la L1 a lo largo de la escolaridad y sobre todo en los cursos superiores, es inferior al propugnado en el modelo canadiense. Según los últimos datos de que se dispone, esto último no supone una merma en la L1 del niño escolarizado en el modelo $\mathrm{D}$ y —en general - los niveles de castellano son comparables a los de sujetos escolarizados en programas no bilingües (A) (J. Sierra e I. Olaziregi, 1989).

El problema del choque de objetivos se presenta ante la diversidad de situaciones que se puedan plantear en la realidad y que obliguen a reunir a sujetos vascoparlantes de origen, otros relativamente vascoparlantes y alumnos castellanoparlantes bajo un mismo modelo D o ante la ausencia 
FIGURA 1
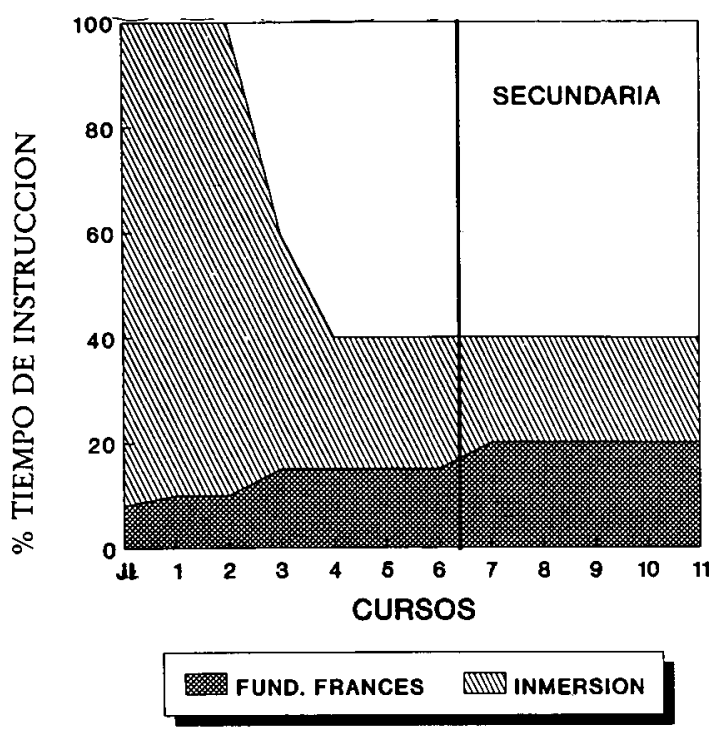

9T. LAMBERT

JI-JAAOIN DE IMTANEIA

Inmersión total temprana en francés.

Tomado de Genesee; Lambert; Holobow. (Con permiso de Infancia y Aprendizaje núm. 33, 1986. (C).

\section{FIGURA 2}

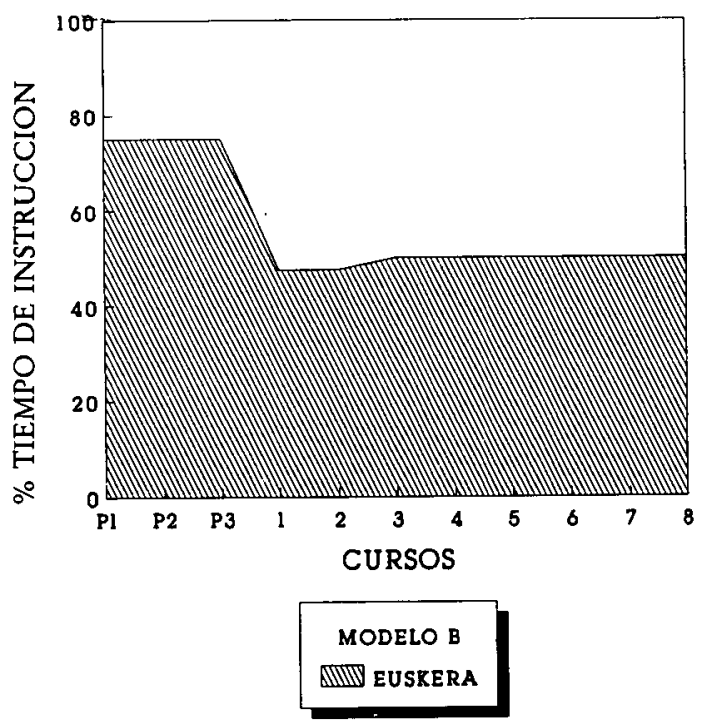

CURSO A CURSO

Modelo B.

(Proporciones de Euskera). 
Figura 3
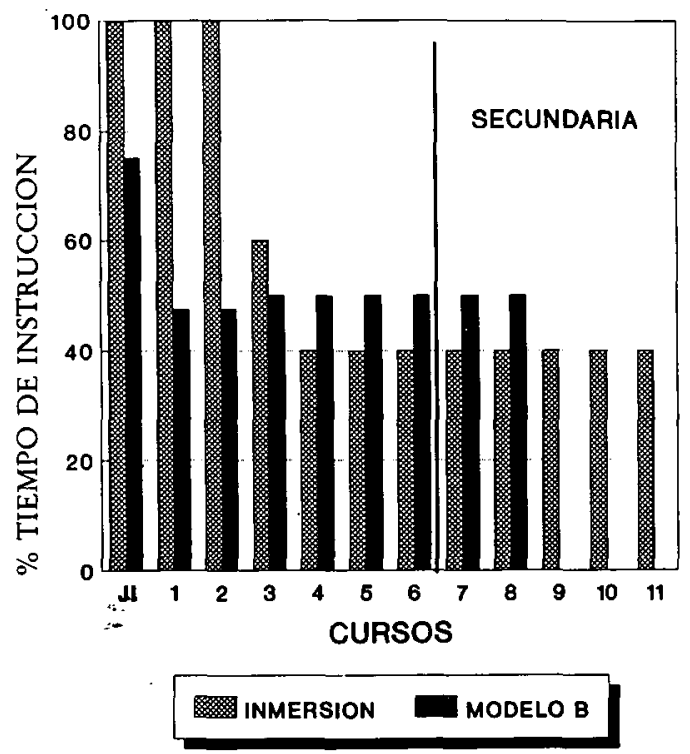

ST. LAMBERT-PAIS VASCO

JPJAAOOIN OE IMPANCIA

Inmersión total temprana en francés, proporciones francés y euskera modelo $B$.

de una metodología específica, elaborada y estructurada que diferencie la adquisición de una L2 de la enseñanza de un vascoparlante en su propia lengua.

\section{Referencias}

ARTigal, J. M. (1989). "La inmersió a Catalunya». Vic: EUMO.

BALKAN, L. (1976). "Los efectos del bilingüismo en las aptitudes intelectuales». Madrid: Marova.

Cummins, J. (1976). *The influence of bilingualism on cognitive growth: A syntesis of research finding and explanatory hypoteses». Toronto en Working Papers on Bilingualism 13. Ed. OISE.

Cummins, J. (1979). "Linguistic Interdependance and the educational development of bilingual children». Review of Educational Research, 49, 222-251.

- (1987). "Immersion programs: Current issues and future direction». En Contemporany educational issues: The canadian mosaic. Toronto Copp Clark.

- (1989). Prólogo. En EIFE 2. La Enseñanza del Euskera: Influencia de los factores. Vitoria-Gasteiz. Servicio Central de Publicaciones del Gobierno Vasco.

Gabiña, J.; Gorostidi, R.; IruRetagolena, R.; Olaziregi, I. y Sierra, J. (1986). «EIFE. La Enseñanza del Euskera: Influencia de los Factores». Servicio Central de Publicaciones. Gobierno Vasco. Vitoria-Gasteiz.

GENESEE, F.; LAMBERT, W. E. y HOLOBOW, N. E. (1986). «La adquisición de una segunda lengua mediante inmersión: El enfoque canadiense». Infancia y Aprendizaje, 33, $27-36$.

HamerS, J. F. y BlANC, M. (1983). «Bilingualité et Bilinguisme». Bruselas: Pierre Mardaga.

LAMBERT, W. E. y TuCKER, G. R. (1972). «Bilingual education of children. The St. Lambert experiment». Rowley: Newbury House.

LAMBERT, W. E. (1975). «Culture and language as factors in learning and education*. En Education of inmigrant students. A. Wolfgang Toronto: OISE.

MACNAmara, J. (1966). "Bilingualism and Primary Education: A study of irish experience». Edinburgh: Edinburgh University Press. 
Olaziregi, I. y SiERra, J. (1987). «PIR-5 Hizkuntz testa. B eta D ereduetako 5-6 urteko haurrentzat». Vitoria-Gasteiz: Servicio Central de Publicaciones del Gobierno Vasco.

SÁNCHEZ, M. P. y DE TEMBLEQUE, R. R. (1986). «La educación bilingüe y el aprendizaje de una segunda lengua: Sus características y principios fundamentalesw. Infancia y Aprendizaje, 33, 3-25.

Sierra, J. y OlAZIREgi, I. (1989). «EIFE 2. La enseñanza del euskera: Influencia de los factores». Vitoria-Gasteiz: Servicio Central de Publicaciones del Gobierno Vasco.

\section{Los modelos de enseñanza bilingüe en el País Vasco. J.} Sierra.

CLE, 1991, 10, 41-55.

\section{Resumen}

Se describen en este artículo los tres modelos de enseñanza bilingüe vigentes en el País Vasco, contrastando su eficacia para la adquisición del euskera en la población escolar.

Datos sobre el autor: El autor es licenciado en psicología y lleva más de diez años trabajando en el campo de la enseñanza bilingüe. Habiendo sido psicólogo de las Ikastolas (escuelas en lengua vasca) de Bizkaia, trabaja actualmente en el Departamento de Educación del Gobierno Vasco en temas relacionados con la investigación sobre Enseñanza Bilingüe.

Dirección: B. Etxepare, 3, 48960 Galdakao (Bizkaia)

(C) De todos los artículos. Deberá solicitarse por escrito autorización de CLE para el uso en forma de facsímil, fotocopia o cualquier otro medio de reproducción. CLE se reserva el derecho de interponer las acciones legales necesarias en aquellos casos en que se contravenga la ley de derechos de autor. 